\title{
Multi-analyte SPR immunoassays for environmental biosensing of pesticides
}

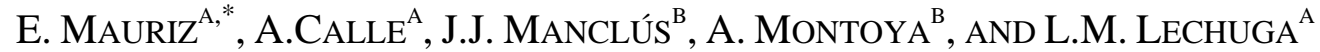

${ }^{a}$ Biosensors Group. Microelectronics National Centre (CNM), CSIC.

Isaac Newton, 8 - 28760 Tres Cantos, Madrid (Spain)

${ }^{b}$ Centro de Investigación e Innovación en Bioingeniería, Universidad Politécnica de

Valencia, Camino de Vera s/n, 46022 Valencia (Spain)

*Corresponding author. Tel.: +34-91-8060700; Fax: +34-91-8060701

E-mail address: elba@imm.cnm.csic.es (E. Mauriz)

\begin{abstract}
Multi-analyte detection of environmentally relevant pesticides is performed by using a two-channelled surface plasmon resonance (SPR) biosensor. The special design of the SPR instrument allows the determination of several analytes (DDT, chlorpyrifos and carbaryl) via different immobilization formats. First, simultaneous pesticide monitoring is possible by flowing chlorpyrifos, carbaryl or DDT samples separately over each channel of the SPR system wherein their corresponding recognition element was previously immobilized. The second approach is based on the multiple and combined immobilization of several analyte recognition elements on the sensing surface of one individual flow cell. In this format, the analysis time for all three pesticides varied from 40 to 60 minutes depending on the number of regeneration cycles. In most of the cases, similar detection limits were attained for the target analyte irrespectively of the assay format,with sensitivity values in the nanogram per litre levels (18-50 ng L ${ }^{-1}$ ). The assay reproducibility was proved through the repeated use of the same sensor surface over more than 200 assay cycles whereas the absence of biosensor response to non-related analytes showed the specificity and reliability of the analysis. The SPR instrument, including optics, electronics and microfluidics, is already commercialised by the company SENSIA, SL.
\end{abstract}

Keywords: DDT; Chlorpyrifos; Carbaryl; SPR immunosensor; Multi-detection; On-line determination; Environmental Monitoring 


\section{Introduction}

The evidence on damages to human health and wildlife from low level pesticide exposures is continually increasing [1]. The lack of monitoring and information on agrochemicals may help their release into the environment via enormous number of urban, agricultural and industrial processes. Because of some of these products can endanger ecosystems and persist for long periods in soils and water resources, environmental-related legislation has focused on the hazard assessment of pesticides to control water quality $[2,3]$. Current toxicity risk assessments are based on single substances whilst people and ecosystems are generally exposed to very complex mixtures. Therefore, it is no longer sufficient to detect one analyte per sample and the evaluation of several compounds at the same time is required.

Chromatographic methods in combination with other detection techniques have been prevalently used for the accurate and sensitive quantification of multiple analytes. However, the need of rapid analyses with minimal sample preparation has led to the development of immunochemical based biosensors for the screening of pesticides in food, agricultural and environmental samples. Immunosensors combine the specificity, sensitivity and low time of response of immunoassays with the capability for automation, miniaturisation and multi-analyte determination.

The main advantages of performing multiple analyses using biosensors are the ability for decreasing the assay time and the possibility of providing more effective comparisons between experimental data. For the monitoring of multiple analytes, biosensors have traditionally adopted different configurations from the microarray technology to a multi-channel performance. One of the first successfully applied strategies was the use of different labels for each analyte. However, the fabrication of arrays has allowed the detection of all analytes using the same tracer by separating immunoreactive molecules on a single surface [4]. A number of array biosensors have been described depending on the selected solid substrate. Among them, the use of glass slides, optical fibers and capillaries are the formats more widely extended in the environmental and clinical field. For instance, array based multi-analyte biosensors developed by Mastichiadis [5], Weller [6], Anderson [7] and Wadkins [8] have reached high sensitivity levels when detecting toxins, DNA and pesticides. Other systems based on total internal reflection fluorescence (TIRF), such as the RIANA and AWACSS systems [9, 10], have also demonstrated the simultaneous detection of three pesticides by spatially resolved surface modification. 
In spite of the significant benefits provided by array-based multi-biosensors, direct detection of the binding event is always preferable since it can be monitored in real-time. SPR biosensors overcome limitations derived from label requirements by measuring changes in refractive index after biomolecule interaction rapidly and in a continuous manner. To date, most multi-analyte SPR biosensors are multi-channel platforms based on imaging systems or spectroscopy technology [11]. SPR imaging multi-analyte biosensors have been proved for the simultaneous monitoring of more than one hundred of measurements by using multiple sensing spots, although with limited resolution. In contrast, SPR spectroscopy-based biosensors consist on the parallel multiplexing of sensing channels and offer better resolution than SPR imaging sensors. In this format, the number of channel is limited to a maximum of 10. Using both SPR multichannel configurations a spatial controlled immobilization of the recognition element is required $[12,13]$.

This work presents an alternative format that exploits a conventional two-channelled SPR configuration to determine up to three analytes on a single sensing surface. The aim of the study is to determine the capability for multi-analysis of an analytical system previously reported for the single detection of three relevant pesticides: DDT, carbaryl and chlorpyrifos [14-16]. With this purpose measurements of the target analytes were carried out using different approaches based on inhibition binding assays.

First, parallel analyte determinations were developed for simultaneous pesticide monitoring by flowing DDT, chlorpyrifos and carbaryl samples separately over each channel of the SPR system. The second approach was based on a multi-analyte immobilization protocol in which two or three analyte derivatives were immobilized on the sensing surface of one individual flow cell. In this format, DDT, chlorpyrifos and carbaryl analyses were carried out using sequential and additive modes [17]. The sequential protocol is based on the performance of the whole analysis cycle of a pesticide, including regeneration, followed by the cycle corresponding to the other two analytes. For the additive protocol the three pesticides are analysed successively and all bound interactants are removed in a single regeneration step. Therefore, the analysis time will depend on the number of regeneration cycles.

Although another existing SPR biosensor has been recently used for multi-analyte detection of sulfonamides [18], this is the first SPR-based multi-sensor applied for the simultaneous detection of pesticides. Our SPR biosensor would allow the simultaneous detection of six analytes using the two channels of the system. In addition, the SPR instrument is portable and works under reversible conditions. These particular features could be used for the unattended on-line monitoring of point source discharges in environmental waters. 


\section{Experimental}

\subsection{Chemicals and immunoreagents}

For SPR immunoassays, all the immunoreagents were prepared at the Centro de Investigación e Innovación en Bioingeniería (Universidad Politécnica de Valencia, Spain). The synthesis of DDT [DDT5, 4-\{4-[1-(4-chlorophenyl)-2,2,2trichloroethyhl]phenyl\}butanoic acid], chlorpyrifos [CN4C, O-Ethyl O-(3,5,6-Trichloro-2-pyridyl) N-(3-Carboxypropyl) Phosphoramido thioate] and carbaryl [CNH, 6-[[1-naphtyloxy)carbonyl]-amino] hexanoic acid] haptens; the preparation of the BSA-DDT5, BSA-CN4C and BSA-CNH conjugates; and the production of anti-DDT monoclonal antibody (MAb) LIB-DDT5.25, anti-carbaryl MAb LIBCNH45 and anti-chlorpyrifos MAb LIB-PO have been previously described in other papers [19-21]. The affinity and specificity of the monoclonal antibodies produced against $p, p$ '-DDT, carbaryl and chlorpyrifos were characterized using either antibody-coated or conjugate-coated ELISA formats.

Standards of DDT and carbaryl were obtained from Dr Ehrenstorfer (Augsburg, Germany) whilst chlorpyrifos standard was from Riedel-de Haën (Seelze, Germany). Analytical standards were prepared as stock solutions of $1 \mathrm{mM}$ in dry dimethylformamide (DMF) and stored at $20^{\circ} \mathrm{C}$. Working standards were prepared daily by serial dilutions in PBST (PBS: $10 \mathrm{mM}$ phosphate-buffered saline solution, $\mathrm{pH} 7.35$, containing $0.05 \%$ of the surfactant Tween- 20).

Common chemicals for the immobilization procedure were purchased from Sigma-Aldrich: mercaptoundecanoic acid; N-Hydroxysuccinimide (NHS) and 1-ethyl-3(-3 dimethyl-aminopropyl) carbodiimide hydrochloride (EDC). Ethanolamine hydrochloride used as blocking agent was provided by Acros Organics. Tween 20 (T20) was obtained from Quantum Appligene and the organic solvents utilized for the gold-coated film cleaning: Ethanol and Acetone were supplied by Merck. The additional compounds Potassium chloride, Sodium chloride, di-Sodium Hydrogen Phosphate and Potassium di-Hydrogen Phosphate, used for the preparation of 1x PBS $10 \mathrm{mM}$, were provided by Panreac.

\subsection{SPR instrumentation and sensor chip preparation}

Multi-analyte determination of the target pesticides was carried out by means of the $\beta$-SPR sensor system already commercialised by the company SENSIA S.L. (Spain). The configuration of the SPR device has been reported in previous papers [14-16] (Fig.1). Briefly, it works in the 
Kretschmann configuration to achieve the resonant condition by using a prism coupler structure. A laser beam emitting at $670 \mathrm{~nm}$ from a $3 \mathrm{~mW}$ laser diode source is divided into two equal beams to allow two-channelled simultaneous measurements onto the gold-coated film used as sensing surface. For monitoring binding events in real time, SPR measurements are performed at a fixed angle of incidence. Variations of the refractive index corresponding to shifts in mass at the sensing surface are detected as changes in the reflected light intensity by a multielement photodiode. Finally, the SPR signal is amplified and converted to a digital signal.

The flow delivery system, including two flow cells (300 nL), a peristaltic pump and two diaphragm pumps for loading the sample, is completely integrated into the $\beta$-SPR platform. A precise volume of sample (220 $\mu \mathrm{L})$ is pumped over the SPR sensor chip at a constant speed of $30 \mu \mathrm{L} \mathrm{min}{ }^{-1}$ whilst a continuous flow of buffer or distilled water is delivered onto the sensor surface. The complete multi-assay for three analytes comprises 40-60 minutes, depending on the number of regeneration cycles.

For SPR measurements, microscope cover glass slides $(10 \times 10 \times 0.15 \mathrm{~mm})$ coated with gold at $50 \mathrm{~nm}$ were used as sensor chips. The sensing surface preparation involved an exhaustive cleaning procedure including the use of organic solvents and the gold slide dipping in a freshly prepared piranha solution $\left(\mathrm{H}_{2} \mathrm{SO}_{4} / \mathrm{H}_{2} \mathrm{O}_{2}, 3: 1\right)$. The cleaning treatment is completed with the rinsing and ultrasonicating of the gold layer in distilled water. Sensor chips are placed into the biosensor system by coupling the gold film side to the flow cells and the subsequent adhering of the prism via a matching oil with the same refractive index $(\mathrm{n}=1.52)$.

\subsection{Immobilization procedure}

The stability of the multi- analysis was assured via the formation of a self-assembled monolayer (SAM) of alkanethiol onto the gold layer, as already reported [14-16]. Biomolecule immobilization was accomplished by attaching amine groups of the recognition element to carboxylic terminal groups of the alkanethiol monolayer through a carbodiimide linkage. The biolayer successfully formed is chemically resistant to regeneration agents allowing reliable measurements during a large number of assay cycles.

For the SAM formation, mercaptoundecaonoic acid at $0.05 \mathrm{mM}$ in ethanol was flowed over the gold sensing layer. The activation of the alkanethiol carboxylic groups to a stable intermediate (N-hydroxysuccinimide ester) was accomplished using a mixed solution of EDC/NHS (0.2/0.05M in distilled water) [22]. In this state, the modified sensor surface is easily available 
for the amine groups of the BSA-hapten conjugate used as recognition element. To avoid both non-specific binding and multilayers of non-covalently bound conjugates, a solution of ethanolamine 1M, pH 8.5 was pumped over the sensing surface so that all remaining unreacted NHS-esters could be deactivated.

The use of the modified sensing layer for a multi-analyte purpose involved the simultaneous immobilization of hapten carrier conjugates corresponding to different analytes in the same surface. Because of the difference in hapten to protein molar ratio (MR) the optimum concentration for each conjugate should be carefully determined. The possibility of applying several combined immobilizations for the target conjugates is described in detail below.

\subsection{SPR immunoassay protocols}

SPR detection of small size pesticides was based on the performance of binding inhibition tests wherein mixtures of different analyte concentrations and a fixed antibody dilution are flowed over a sensing layer modified with the hapten derivative of the target analyte [23]. Since the antibody binding to the immobilized hapten is inhibited by the presence of analyte, higher analyte concentrations will yield diminishing biosensor responses.

Using this format, serial dilutions of DDT (0.004-354 $\left.\mu \mathrm{g} \mathrm{L}{ }^{-1}\right)$, chlorpyrifos (0.004-350.6 $\left.\mu \mathrm{g} \mathrm{L}^{-1}\right)$ and carbaryl (0.002-2000 $\left.\mu \mathrm{gL}^{-1}\right)$ were prepared in PBST 1x from their respective stock solutions in DMF. Standard dilutions were mixed with a fixed concentration of the monoclonal antibody for each specific analyte $(1: 1 ; \mathrm{v} / \mathrm{v})$. The final dilutions were incubated for 10 minutes at room temperature before being pumped over the sensing surface. Monitoring of the antibody binding to the immobilized hapten is performed in real-time. The continuous analysis of samples was achieved by regenerating the sensing surface with $0.1 \mathrm{M} \mathrm{HCl}$ after each antibody-hapten association. The same protocol was carried out in single or multi-analyte immobilization formats with the only significant difference of the number of regeneration cycles.

\subsection{Data analysis}

The reliability of the generated surface to reproduce measurements was assured determining, three mixed analyte-antibody samples for each concentration of DDT, chlorpyrifos and carbaryl standards. Normalized standard curves were obtained by plotting reflectance (V) against the 
logarithm of chlorpyrifos concentration. The experimental points were fitted to the fourparameter logistic equation:

$$
\mathrm{y}=\left\{\mathrm{D}+(\mathrm{A}-\mathrm{D}) /\left[1+(\mathrm{x} / \mathrm{C})^{\mathrm{B}}\right]\right\}
$$

where $A$ is the asymptotic maximum (maximum SPR signal in absence of analyte, $A_{\max }$ ), $B$ is the curve slope at the inflection point (related to the analyte concentration giving 50\% inhibition of $A_{\max }: C, I_{50}$ ) and $D$ is the asymptotic minimum (background signal). The standard curves for DDT, carbaryl and chlorpyrifos were obtained by averaging three individual standard curves and normalized by expressing the SPR signal ( $\left.\mathrm{SPR}_{\text {signal }}\right)$ of each standard point as the percentage of the maximum response $\left[100 \mathrm{x}\left(\mathrm{SPR}_{\text {signal }} / \mathrm{SPR}_{\text {signal,max }}\right)\right]$.

\section{Results}

\subsection{Characterization of the immobilization protocols}

The particular design of our SPR device allows the simultaneous monitoring of binding events ocurring separately in its two channels. Therefore, two different strategies can be followed to detect the target pesticides by using single or multiple hapten conjugate immobilization formats. For the single immobilization approach, hapten-antibody interactions corresponding to only one analyte can be determined in each channel at the same time. In this format, the multi-biosensor capability could be proved for a maximum of two analytes. The second approach relies on the simultaneous immobilization of mixtures of two or three hapten conjugates on the modified surface of one individual flow cell. The simplicity and reliability of this method is significant with respect to other multi-assay transducers since the spatially resolved modification of the surface is not needed. The only specific requirement is that a sample containing different analyte derivatives at appropriate concentrations flows over the activated alkanethiol sensor layer.

\subsubsection{Single derivative immobilization}

The most straightforward procedure for detecting two analytes simultaneously is by individual immobilization of one analyte derivative on each flow cell of the sensor surface. For measuring a single analyte, the SPR sensor surface was modified with one hapten by covalent bond to the carbodiimide activated gold substrate. The optimized conditions for BSA-DDT5, BSA-CNH and BSA-CN4C single immobilization has been described in previous papers [14-16, 24]. Following, this format DDT, carbaryl and chlorpyrifos conjugates were prepared in PBST to a 
final concentration of $10 \mu \mathrm{g} \mathrm{mL}^{-1}$. The performance of single analyte measurements were carried out by flowing standard solutions separately over the channel in which its corresponding derivative had been previously immobilized. Fig. 2 shows binding inhibition tests performed for the simultaneous detection of DDT and carbaryl over a sensor surface with their corresponding haptens immobilized in each flow cell .

\subsubsection{Multiple derivative immobilizations}

An alternative to parallel biosensing is offered by immobilization of multiple derivatives in the same sensor surface. Using this format, the same substrate can be modified with groups of two or three derivatives depending on the combination selected for the analysis. In all cases, SPR signals to hapten-antibody bindings should be similar to those attained in single-conjugate immobilization formats, thus indicating similar assay sensitivity in both formats. Furthermore, the signal intensity may assure clear differentiation between diminishing analyte concentrations during the performance of binding inhibition tests. For SPR measurements, the minimum allowable signal for the blank (analyte-free) sample was set at 0.10 a.u. (arbitrary units). The SPR response was evaluated through successive antibody injections at the concentration used for single-analyte measurements, i.e., $2.5 \mu \mathrm{g} \mathrm{mL} \mathrm{m}^{-1}$ for anti-DDT MAb (LIB-DDT5.25); $2 \mu \mathrm{g}$ $\mathrm{mL}^{-1}$ for anti-carbaryl MAb (LIB-CNH45); and $1 \mu \mathrm{g} \mathrm{mL}^{-1}$ for anti-chlorpyrifos MAb (LIB-PO).

The simultaneous immobilization of three derivatives in the same surface required several combinations of conjugate concentrations. As Table 1 shows, the optimal combination of multiconjugate concentrations was obtained for 15, 10 and $5 \mu \mathrm{g} \mathrm{mL}{ }^{-1}$ dilutions of DDT, carbaryl and chlorpyrifos conjugates. Attending to these results, the use of either balanced or higher conjugate concentrations did not ensure similar immunosensor responses for all hapten-antibody interactions. The key factor for mixed immobilizations was the distribution and accessibility of haptens along the surface irrespectively of the hapten to protein molar ratio of each individual conjugate.

The possibilities available for binary mixture immobilizations are shown in Table 2. Following the criteria previously described, most favourable combined immobilizations were found for: 20 and $7.5 \mu \mathrm{g} \mathrm{mL}^{-1}$ of BSA-DDT5 and BSA-CNH mixture; 20 and $10 \mu \mathrm{g} \mathrm{mL}^{-1}$ of BSA-DDT5 and BSA-CN4C conjugates; and 10 and $5 \mu \mathrm{g} \mathrm{mL}^{-1}$ of BSA-CNH and BSA-CN4C final solution. 


\subsection{Optimization of multi-immunoassay formats}

The performance of binding inhibition tests over multi-conjugate immobilized surfaces involves the possibility of detecting several analytes from different approaches. The first ideal strategy consists in the simultaneous determination of all three or two target analytes mixed in the same sample. This format would require the development of complicated data interpretation designs in order to assign the hapten-antibody signal corresponding to each analyte. In addition, this method could only be applied for qualitative analyses since the assay accuracy depends on the system considered for signal modulation.

For this reason, multi-analyte determination of two or three analytes in the same substrate was carried out using two other distinct modes based on the sequential or additive analyses of samples.

The sequential protocol allowed the attainment of calibration standard curves in an ordered sequence in which serial dilutions of one of the target analytes will be followed by the corresponding to the other two pesticides. The stability and reproducibility of the immunosensor response was not affected by the order of antibody injections. An assay cycle including each antibody interaction and their corresponding regeneration cycles takes 60 minutes (Fig.2 ba)). This format could be extended to the parallel detection of a single pesticide on one channel whilst monitoring the other two analytes sequentially on the second one. Since an individual assay cycle is completed in 20 minutes the analysis time for the extended format would not vary.

On the other hand, the additive protocol comprises the successive injection of the three antibodies without regenerating between them. Only a final regeneration cycle is included after the latter antibody injection. As Fig.2b) shows, the analysis time can be reduced to 40 minutes. However, in this format, the SPR signal provided by each antibody is highly related to the order of injection and, consequently, the six possible existing antibody sequences have to be evaluated (see Table 3). In addition, the capability for surface regeneration also depends on the order of injections and a compromise between signal intensity and the number of regeneration cycles have to be adopted. According to these conditions, the optimal antibody sequence was obtained for anti-DDT MAb, followed by chlorpyrifos and carbaryl MAbs.

\subsection{Sensitivity of single and multi-assay formats}


The assay sensitivity was estimated from seven-point standard curves run in triplicate. The SPR signal was inversely proportional to the analyte concentration in the analyte-antibody mixture, and measurements were fitted to a sigmoidal equation. Limits of detection (LODs) were experimentally determined as the analyte concentration giving a $10 \%$ inhibition of the maximal SPR signal. The linear working ranges were also defined as the analyte concentration causing a 20-80\% decrease of the blank signal.

The multi-surface sensor with immobilized derivatives of DDT, carbaryl and chlorpyrifos is based on a previously calibrated single-analyte format [14-16]. In comparison with the results obtained using single-conjugate sensor surfaces, multi-analyte measurements in the sequential mode shown similar sensitivity values (see Fig. 3 and Table 4).

Limits of detection of 32 and $18 \mathrm{ng} \mathrm{L}^{-1}$,and $\mathrm{I}_{50}$ values of 1.06 and $0.44 \mu \mathrm{g} \mathrm{\textrm {L } ^ { - 1 }}$ were attained for DDT individual and simultaneous immobilization formats, respectively. Chlorpyrifos inhibition binding curves also reached an extremely similar linearity, and low detection limits (54-52 ng L ${ }^{1}$ ) and $I_{50}(1.96-1.76) \mu g L^{-1}$ values for both assay protocols. Finally, the assay sensitivity obtained for carbaryl single and multi-analyte approaches were slightly different for the $\mathrm{I}_{50}$ values (3.11-1.23 $\mu \mathrm{g} \mathrm{L}^{-1}$ ) whilst a significant improvement of the detection limit was observed when comparing the multi-analyte format $\left(0.05 \mu \mathrm{g} \mathrm{L}^{-1}\right)$ to the single analyte one $\left(1.41 \mu \mathrm{g} \mathrm{L}{ }^{-1}\right)$. This means that the most favourable conditions for carbaryl conjugate immobilization are found in a multi-conjugate format with three immobilized derivatives. Further research on the combined immobilization of carbaryl conjugate with proteins of similar molecular weight could improve its performance in single immobilization protocols. Most relative standard deviation (RSD) values were in the 2-10\% range. Taking in account that variability values around $20 \%$ are acceptable for typical immunoassays, this biosensor could be used for both, quantitative analysis and early detection of environmental pollutants.

With respect to the multi-analysis in the additive mode, inhibition curves could only be obtained for the antibody injected in the second place: anti-chlorpyrifos MAb LIB-PO. In this case, sensitivity values were in the same range to those achieved by individual and simultaneous immobilization formats ( $\mathrm{LOD}=0.03 \mu \mathrm{g} \mathrm{L}^{-1}$ ). On the contrary, binding inhibition tests with the rest of analytes (DDT and carbaryl) could not be successfully performed due to the early inhibition of the SPR signal for low concentrations of analyte, thus decreasing the sensitivity of the assay. As Fig.2b) shows, this fact could be related to the presence of bound antibodies from the previous cycle, because of only partially regenerated biosurfaces. 


\subsection{Specificity of multi-immunoassays}

The evaluation of the multi-surface sensor to recognize specifically free-antibodies present in the sample was carried out by flowing analyte-antibody mixtures containing the other nonrelated analytes. For instance, serial dilutions prepared for the detection of DDT in the 0.004$354 \mu \mathrm{g} \mathrm{L}^{-1}$ range were first mixed with its specific MAb (LIB-DDT5.25), as commonly used for binding inhibition tests, and then fortified with chlorpyrifos and carbaryl standards. In this case, DDT would be considered as the specific analyte whilst chlorpyrifos and carbaryl as the crossreactants compounds. The same procedure is applied for carbaryl and chlorpyrifos serial dilutions, although mixing with their non-specific analytes in each case.

The specificity of the assay was studied in the sequential mode at three concentration levels $(0.5,5$ and $50 \mathrm{nM})$ of the specific analyte and at a fixed concentration $(500 \mathrm{nM})$ of the other two compounds. Table 5 shows the comparison between concentration values in the presence and absence of cross-reactants. In most of the assays, only slight deviations from the analytical standard values were observed, displaying similar sensitivity values in both formats.

According to these results, the immunosensor response was not affected by the presence of other analytes in the same sample. And consequently, multiple immobilization protocols would assure a suitable route for the reaction of complementary interactants without interference of non-related compounds.

\section{Conclusions}

The performance of a SPR immunosensor system was evaluated for the simultaneous detection of priority substances within the main groups of pesticides: DDT (organochlorine), chlorpyrifos (organophosphorus) and carbaryl (carbamate) insecticides. Multi-assays were carried out by using its particular two-channelled configuration for single and multiple immobilizations of derivatives of the target analytes.

The immobilization of a single derivative allowed the simultaneous monitoring of antibody interactions occurring separately on each individual flow-cell. On the contrary, multi-surface sensors with several immobilized derivatives involved the use of one independent flow cell for the analysis of two or three analytes. The modification of the same substrate with groups of two 
derivatives was exploited for all the existing combinations of binary mixtures. In this format, analyte determinations were carried out using sequential or additive modes depending on the number of regeneration cycles performed. The results show that the additive analysis of samples had no advantages over the sequential protocol since regeneration of the activated surface was not successfully achieved. Therefore, all multi-analyte measurements were accomplished in the sequential mode.

The comparison between single- and multi-assay sensitivity values showed similar detection limits irrespectively of the analysis format. In the majority of approaches, these limits were within the maximum allowable concentrations prescribed by European Directives for single pesticides in drinking water. The lowest sensitivity values were found for DDT and chlorpyrifos determinations in both single and multi-immobilization protocols, with detection limits between 18 and 54 nanograms per litre levels. Multi-analyte sensitivity for carbaryl determinations was also comparable to DDT and chlorpyrifos analyses, showing a highly sensitive detection in multi-surfaces with three immobilized derivatives ( $\mathrm{LOD}=50 \mathrm{ng} \mathrm{\textrm {L } ^ { - 1 }}$ ). However, further investigation will be performed to improve the limits of detection of single and binary immobilization formats.

The influence of cross-reactants was also evaluated by flowing samples containing all three analytes. The immunosensor response deviation from the expected signal in the absence of nonspecifics analytes was negligible, showing the specificity of the multi-surface to recognize only the target analyte.

The SPR multi-surface approach has shown to be effective for determining the target analytes in a highly sensitive, simple and rapid manner, without the need of labelling biomolecules or spatially resolved surface modification. These are inherent advantages over chromatographic and fluorescence-based multi-biosensors. In addition, this method could be extended to the detection of more than three analytes by simply modifying the sensing surface with a higher number of compounds. This SPR biosensor has also been tested for the detection of environmental waters showing comparable values with chromatographic methods used as reference. The multi-biosensing system offers a versatile, regenerable and robust method for the environmental monitoring of DDT, carbaryl, chlorpyrifos and other organic pollutants, from endocrine disrupters to pharmaceuticals, whenever immunoreagents are available .References

1. European Environmental Agency (EEA), 1998. Chemicals in the European Environment: Low Doses High Stakes. The EEA and UNEP Annual Message 2 on the State of Europes's Environment. 
2. 2000/60/EC, 2000. Directive 2000/60/EC of the European Parliament and of the Council of 23 October 2000 establishing a framework for Community action in the field of water policy. Off. J. Eur. Commun. L327, 1-72

3. 98/83/EC, 1998. Council Directive (98/83/EC) of 3 November 1998 relating to the water quality of water intended for human consumption. Off. J. Eur. Commun. L330, $32-54$

4. Rowe-Taitt C, Hazzard J, Hoffman KE, Cras JJ, Golden JP, Ligler FS (2000) Biosens. Bioelectron 15:579-589

5. Mastichiadis C, Kabakos E, Christofidis I, Kouparis MA, Winletts C, Misiakos K, (2002) Anal Chem 74 : 6064-6072

6. Weller MG (2005) Anal Bioanal Chem $381: 41-43$

7. Anderson GP, King KD, Gaffney KL, Johnson LH (2000) Biosens Bioelectron 14: 771777

8. Wadkins RM, Golden JP, Pritsiolas LM, Ligler FS (1997) Biosens Bioelectron 13: 407415

9. Proll G, Tschmelak J, Gauglitz G (2005) 381: 61-63

10. Rodriguez-Mozaz S, Lopez de Alda MJ, Barceló D (2006) Talanta 69: 377-384

11. Homola J, Vaisocherova H, Dostálek J,Piliarik M (2005) Methods 37: 26-36

12. Wegner GJ, Alastair WW, Lee HJ, Corner E, Saeki T, Fang S, Corn RM (2004) Anal Chem 76: 5677-5684

13. O’Brien MJ, Pérez-Luna VH, Brueck SRJ, López GP (2001) Biosens Bioelectron 16: 97-108

14. Mauriz E, Calle A, Manclús JJ, Montoya A, Hildebrandt A, Barceló D, Lechuga LM Biosens Bioelectron, in press

15. Mauriz E, Calle A, Abad A, Montoya A, Hildebrandt A, Barceló D, Lechuga LM (2006) Biosens Bioelectron 21: 2129-2136

16. Mauriz E, Calle A, Manclús JJ, Montoya A, Quitana J, Lechuga LM (2006) Anal Chim Acta 561: 40-47 
17. Gonzalez-Martínez MA, Puchades R, Maquieira A, (2001) Anal Chem 73: 4326-4332

18. Bienenmann-Ploum M, Korpimäki T, Haasnoot W, Kohen, F (2005) Anal Chim Acta 529: $115-122$

19. Abad A, Manclús JJ, Mojarrad F, Mercader JV, Miranda MA, Primo J, Guardiola V, Montoya A (1997) J Agr Food Chem 45: 3694-372

20. Abad A, Primo J, Montoya A, (1997) J Agr Food Chem 45: 1486-1494

21. Manclús, JJ, Primo J, Montoya A, (1996) J Agr Food Chem 44: 4052-4062

22. Grabarek Z, Gergely J, (1990) Anal Biochem 185: 131-135

23. Baeumner J Anal Bioanal Chem (2003) 377: 434-445

24. Mauriz E, Calle A, Manclús JJ, Montoya A, Lechuga LM Sens Actuactors, B, Chem, in press. 


\section{Figures}

a)

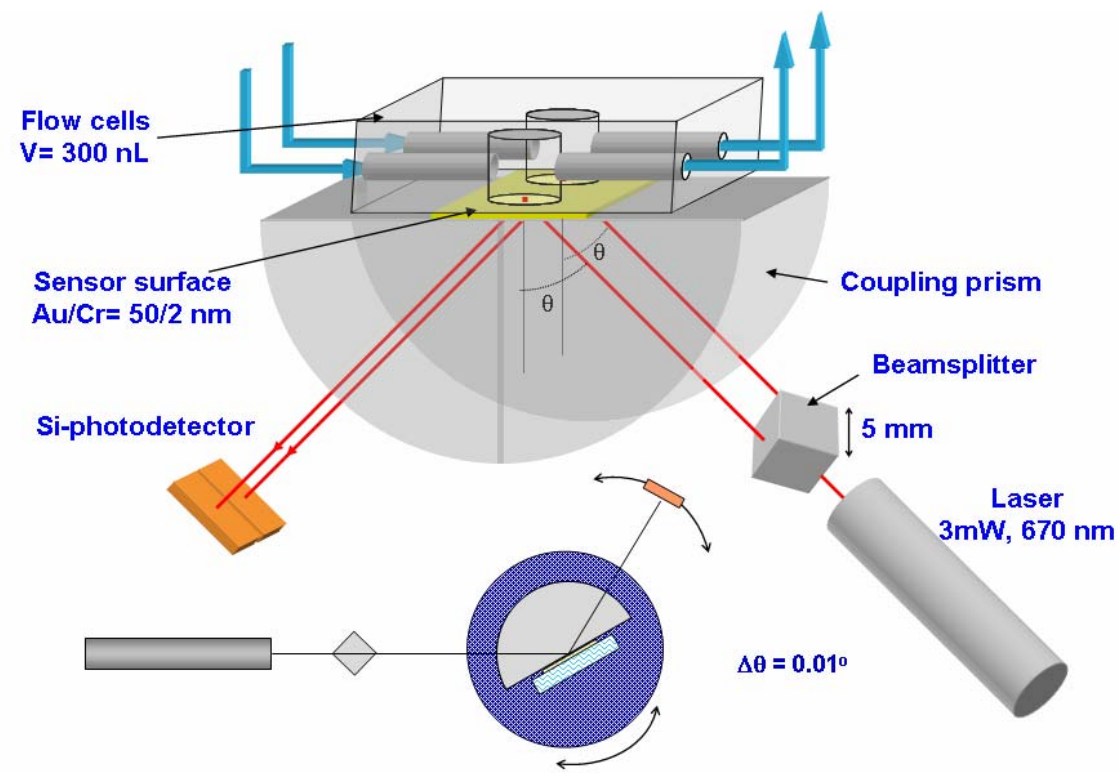

b)

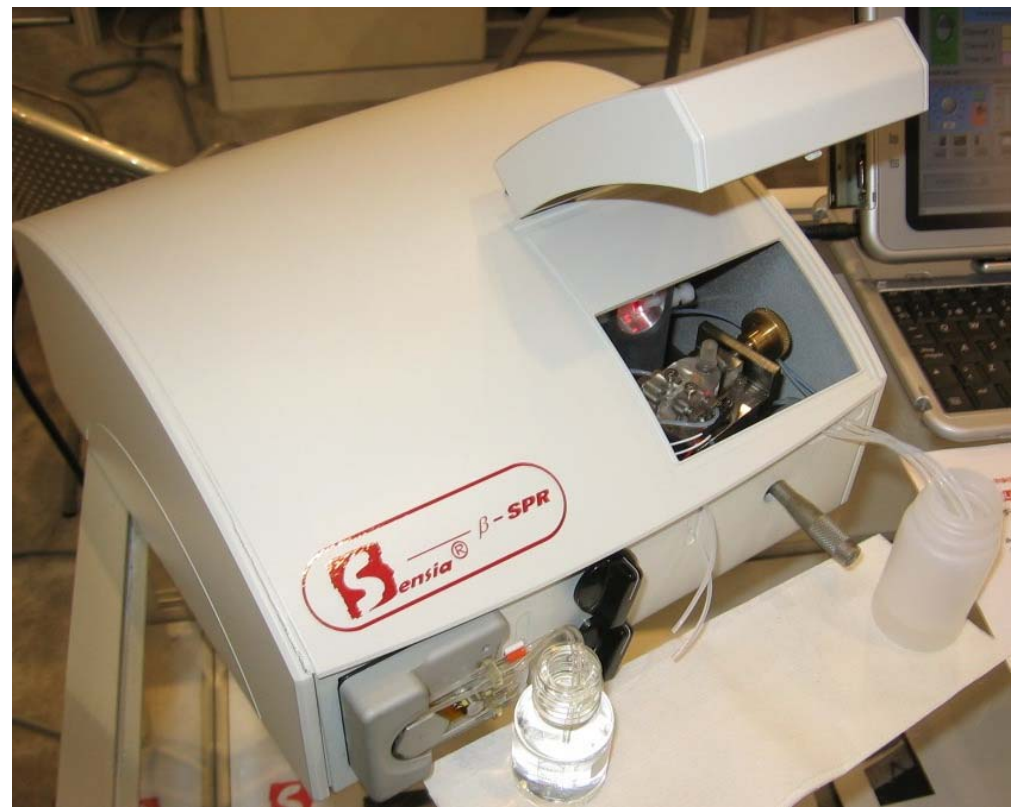

Fig.1. Portable $\beta$-SPR sensor system from SENSIA, S.L., including sensor, optics, electronics and flow delivery system: a) scheme of the SPR device and b) commercial $\beta$-SPR platform 
a)

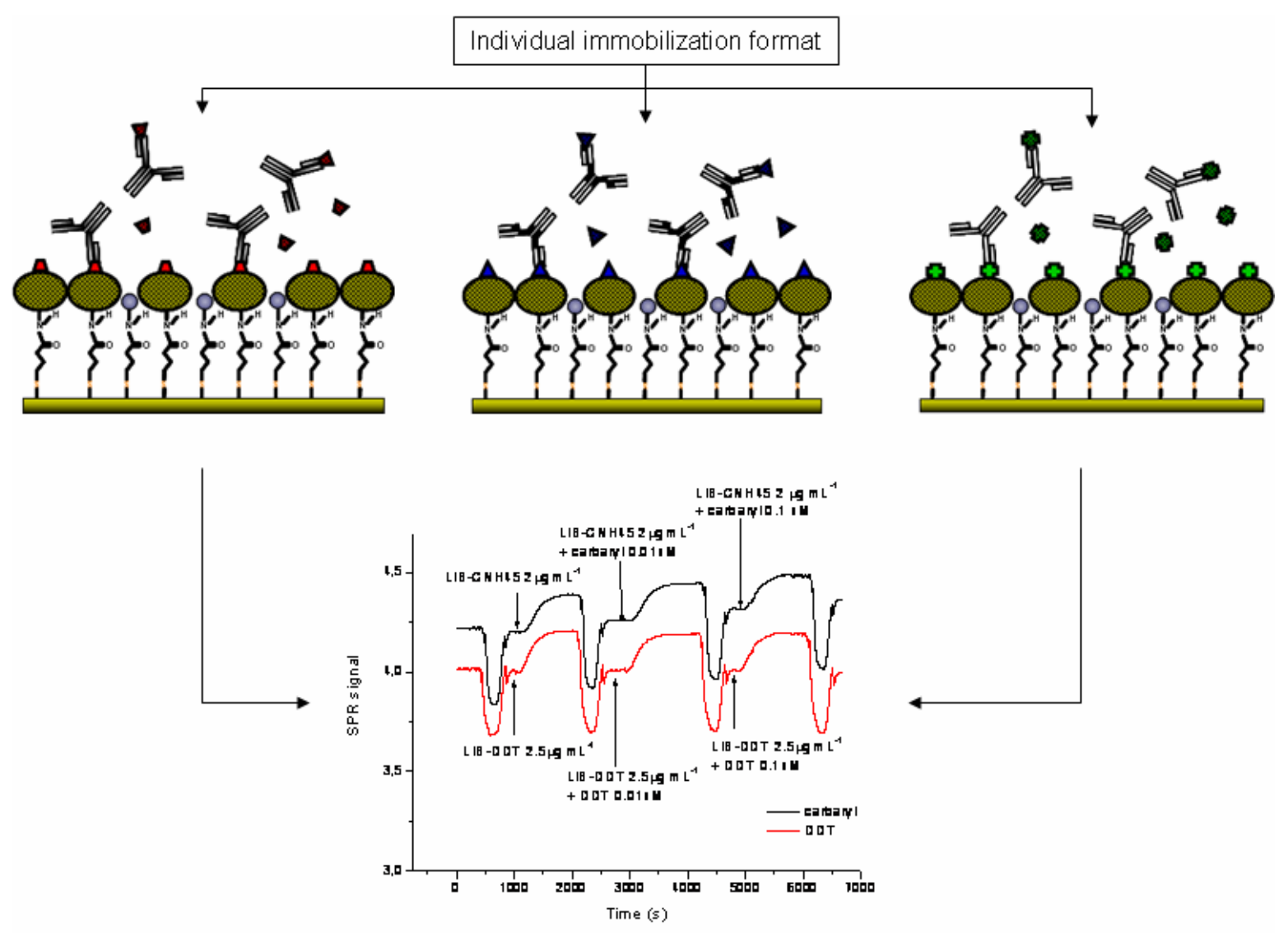


b)

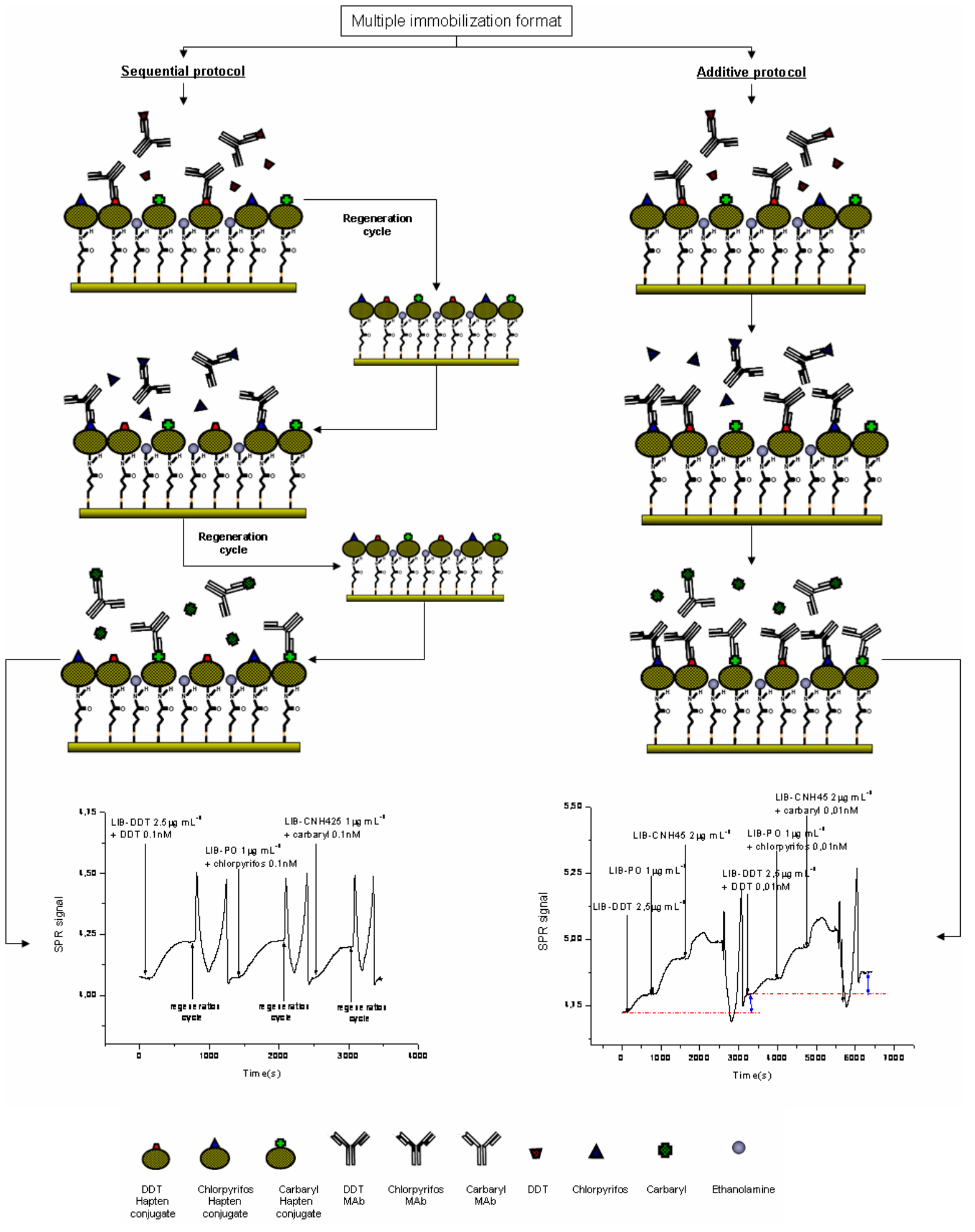

Fig.2. Schematic representation and SPR response to binding inhibition tests using different analytical modes: a) individual and b) multiple hapten conjugate immobililzation protocols. 
a)

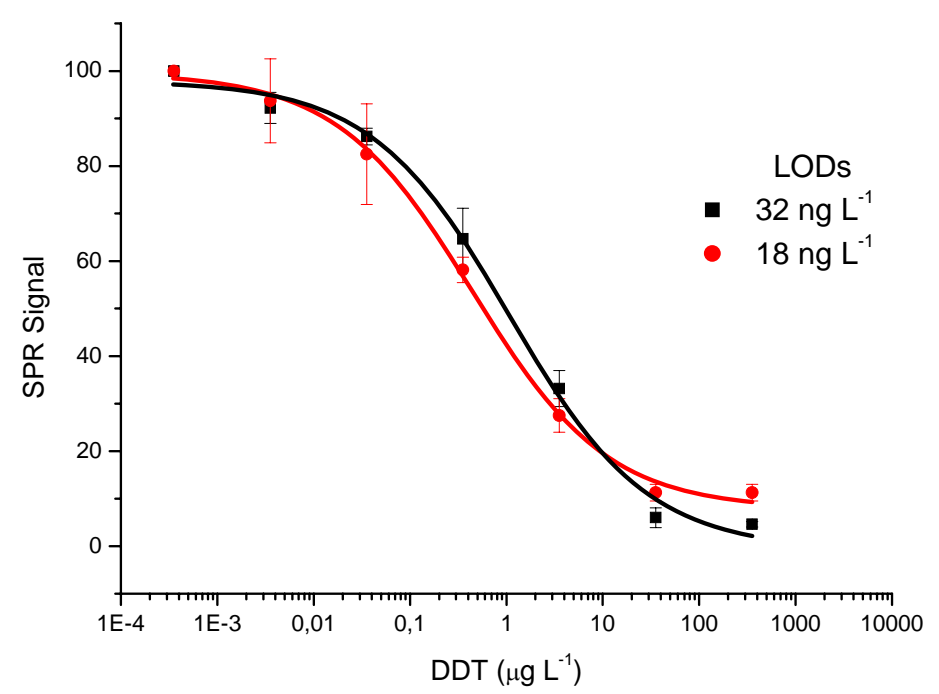

b)

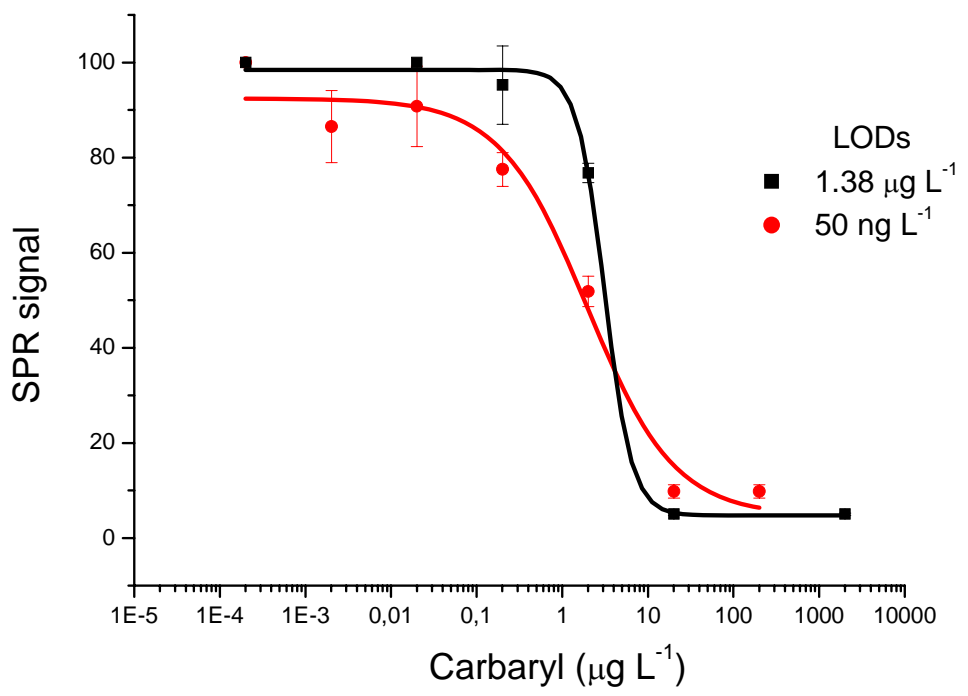


c)

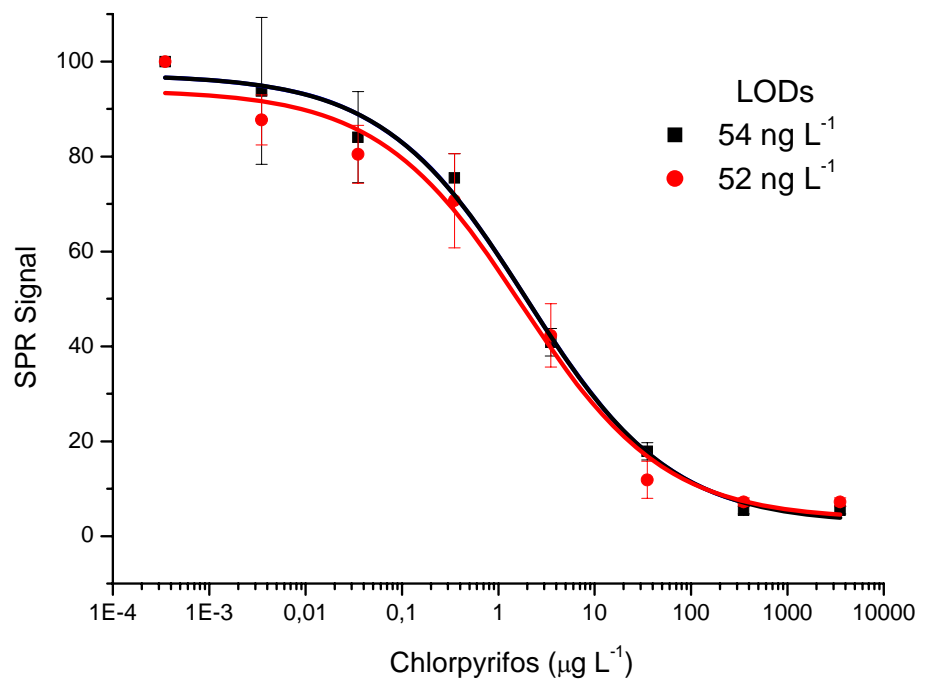

Fig. 3. Standard calibration curves for: a) DDT, b) chlorpyrifos and c) carbaryl single ( $\boldsymbol{\square}$ ) and multi-assay $\left(^{\circ}\right.$ ) (three derivatives) immobilization formats. Meausurements were done in triplicate. 
Table 1

Evaluation of SPR signals for hapten-antibody interactions by using several combinations of the multi-conjugate mixtures of BSA-DDT5, BSA-CNH45 and BSA-CN4C.

\begin{tabular}{|c|c|c|c|c|c|}
\hline \multirow{2}{*}{$\begin{array}{l}\text { SPR Signal } \\
\quad \text { (a.u.) }\end{array}$} & \multicolumn{5}{|c|}{$\begin{array}{c}\text { BSA-DDT5/BSA-CNH /BSA-CN4C } \\
\text { (final concentration, } \mu \mathrm{g} \mathrm{mL}^{-1} \text { ) }\end{array}$} \\
\hline & $10 / 10 / 10$ & $40 / 20 / 20$ & $20 / 10 / 10$ & $40 / 20 / 10$ & $15 / 10 / 5$ \\
\hline $\begin{array}{l}\text { LIB-DDT5.25 } \\
\left(2,5 \mu \mathrm{g} \mathrm{mL}^{-1}\right)\end{array}$ & 0.08 & 0.19 & 0.15 & 0.09 & 0.12 \\
\hline $\begin{array}{l}\text { LIB-CNH45 } \\
\left(2 \mu \mathrm{g} \mathrm{mL}^{-1}\right)\end{array}$ & 0.28 & $\begin{array}{c}0.10 \\
\left(3 \mu \mathrm{gL}^{-1}\right)\end{array}$ & $\begin{array}{c}0.10 \\
\left(3 \mu \mathrm{gL}^{-1}\right)\end{array}$ & 0.07 & 0.15 \\
\hline $\begin{array}{l}\text { LIB-PO } \\
\left(1 \mu \mathrm{g} \mathrm{mL}{ }^{-1}\right)\end{array}$ & 0.25 & 0.29 & 0.24 & 0.19 & 0,16 \\
\hline
\end{tabular}


Table 2

Evaluation of SPR signals for hapten-antibody interactions by using several combinations of binary conjugate mixtures of BSA-DDT5, BSA-CNH45 and BSA-CN4C.

\begin{tabular}{lcccccccc}
\hline & \multicolumn{3}{c}{ BSADDT5/ } \\
Señal SPR & \multicolumn{3}{c}{ BSA-CNH } & \multicolumn{3}{c}{ BSADDT5/BSA-CN4C } & BSACNH/ \\
(a.u..) & $5 / 5$ & $20 / 10$ & $20 / 7,5$ & $15 / 7,5$ & $15 / 10$ & $10 / 5$ & $20 / 10$ & $10 / 5$ \\
nIB-DDT5.25 & 0.08 & 0.10 & $\mathbf{0 . 2 0}$ & 0.11 & 0.08 & 0.10 & $\mathbf{0 . 2 2}$ & - \\
LIB-CNH45 & 0.22 & 0.14 & $\mathbf{0 . 1 6}$ & - & - & - & - & $\mathbf{0 . 1 2}$ \\
LIB-PO & & - & - & 0.08 & 0.25 & 0.15 & $\mathbf{0 . 1 6}$ & $\mathbf{0 . 1 6}$ \\
\hline
\end{tabular}


Table 3

Analysis of variations of SPR signal for antibody interaction as a function of the order

\begin{tabular}{|c|c|c|c|c||c|c|}
\hline \multicolumn{2}{|c|}{} & \multicolumn{1}{|c|}{0,05} & 0,05 & \multicolumn{1}{c|}{0,09} & \multicolumn{2}{|c|}{} \\
\cline { 3 - 8 } & 0,08 & 0,04 & \multicolumn{1}{c|}{0,08} & \multicolumn{2}{c|}{} \\
\hline 0.06 & 0.11 & LIB-DDT5.25 & LIB-CNH 45 & LIB-PO & 0.11 & 0.09 \\
\hline 0.10 & 0.11 & LIB-PO & & LIB-DDT5.25 & 0.11 & 0.07 \\
\hline 0.10 & 0.15 & LIB-CNH 45 & & LIB-CNH 45 & 0.06 & 0.06 \\
\hline
\end{tabular}

of injection by performing in the additive mode. 
Table 4

Analytical sensitivity values for DDT, carbaryl and chlorpyrifos using single and multiconjugate formats

\begin{tabular}{|c|c|c|c|c|c|c|}
\hline & \multicolumn{3}{|c|}{ Single-analyte assays } & \multicolumn{3}{c|}{ Multi- conjugate combinations } \\
\cline { 2 - 7 } & $\begin{array}{c}\text { BSA- } \\
\text { DDT5 }\end{array}$ & BSA-CNH & BSA-CN4C & \multicolumn{2}{c|}{ BSA-DDT5/BSA-CNH/BSA-CN4C } \\
\cline { 2 - 7 } & DDT & Carbaryl & Chlorpyrifos & DDT & Carbaryl & Chlorpyrifos \\
\hline $\begin{array}{c}\mathrm{I}_{50} \\
\left(\mu \mathrm{g} \mathrm{L}^{-1}\right)\end{array}$ & 1.06 & 3.11 & 1.96 & 0.44 & 1.23 & 1.76 \\
\hline $\begin{array}{c}\left.\mathrm{LOD}^{-1}\right) \\
\left(\mu \mathrm{g} \mathrm{L}^{-1}\right)\end{array}$ & 0.032 & 1.38 & 0.054 & 0.018 & 0.050 & 0.052 \\
\hline \hline
\end{tabular}

\begin{tabular}{|c|c|c|c|c|c|c|}
\hline & \multicolumn{6}{|c|}{ Binary mixture combinations } \\
\hline & \multicolumn{2}{|c|}{$\begin{array}{c}\text { BSA-DDT5/ } \\
\text { BSA-CNH }\end{array}$} & \multicolumn{2}{|c|}{$\begin{array}{l}\text { BSA-DDT5/ } \\
\text { BSA-CN4C }\end{array}$} & \multicolumn{2}{|c|}{$\begin{array}{l}\text { BSA-CNH/ } \\
\text { BSA-CN4C }\end{array}$} \\
\hline & DDT & Carbaryl & DDT & Chlorpyrifos & Carbaryl & Chlorpyrifos \\
\hline $\begin{array}{c}\mathrm{I}_{50} \\
\left(\mu \mathrm{g} \mathrm{L}^{-1}\right)\end{array}$ & 2.02 & 2.42 & 0.82 & 0.49 & 2.46 & 1.027 \\
\hline $\begin{array}{c}\text { LOD } \\
\left(\mu \mathrm{g} \mathrm{L}^{-1}\right)\end{array}$ & 0.03 & 1.73 & 0.017 & 0.04 & 0.48 & 0.018 \\
\hline
\end{tabular}




\section{Table 5}

Comparison of analytical values for Standard samples using multi-surfaces with three immobilized derivatives. Measurements were performed in the absence and presence of crossreactants added at different concentrations of specific analyte $(0.5,5$ and $50 \mathrm{nM}$, expressed in

\begin{tabular}{|c|c|c|c|c|c|c|}
\hline & \multicolumn{6}{|c|}{ Serial dilutions } \\
\hline & \multicolumn{2}{|c|}{ DDT } & \multicolumn{2}{|c|}{ Carbaryl } & \multicolumn{2}{|c|}{ Chlorpyrifos } \\
\hline & DDT & $\begin{array}{c}\text { DDT + } \\
\text { cross- } \\
\text { reactants }\end{array}$ & Carbaryl & $\begin{array}{l}\text { Carbaryl } \\
+ \text { cross- } \\
\text { reactants }\end{array}$ & Chlorpyrifos & $\begin{array}{c}\text { Chlorpyrifos } \\
\text { + cross- } \\
\text { reactants }\end{array}$ \\
\hline \multirow{3}{*}{$\begin{array}{l}\text { Concentrations } \\
\left(\mu \mathrm{g} \mathrm{L}^{-1}\right)\end{array}$} & 0.18 & 0.21 & 0.10 & 0.077 & 0.17 & 0.14 \\
\hline & 1.77 & 1.2 & 1.00 & 0.74 & 1.75 & 1.25 \\
\hline & 17.72 & 16.99 & 10.06 & 10.10 & 17.52 & 19.93 \\
\hline $\mathrm{I}_{50}\left(\mu \mathrm{g} \mathrm{L}^{-1}\right)$ & 0.44 & 0.41 & 1.23 & 1.82 & 1.76 & 1.55 \\
\hline $\operatorname{LOD}\left(\mu \mathbf{g ~ L}^{-1}\right)$ & 0.018 & 0.014 & 0.050 & 0.18 & 0.052 & 0.032 \\
\hline
\end{tabular}

$\mu \mathrm{g} \mathrm{L}^{-1}$ for each pesticide). All recoveries were in the $70-120 \%$. 